\title{
Successful Treatment of a Cesarean Scar Ectopic Pregnancy Using a Novel Approach with Oral Mifepristone followed by Trans Cervical Suction Evacuation with Prophylactic Tranexamic Acid: A Case Report
}

Muhunthan $K, M B B S, M S, F R C O G^{1^{*}}$ (D) Anton Jenil Amirthanathan, MBBS, $M D^{2}$ (D), Raguraman S, MBBS, MD, MRCOG ${ }^{1}$ iD and Guruparan $K, M B B S, M D^{1}$ (D)

${ }^{1}$ Department of Obstetrics and Gynecology, Faculty of Medicine, University of Jaffna, Sri Lanka

${ }^{2}$ Department of Radiology, Base Hospital, Point Pedro, Sri Lanka

*Corresponding author: Muhunthan K, Department of Obstetrics and Gynecology, Faculty of Medicine, University of Jaffna, Postcode - 40000, Sri Lanka, Tel: +947-774-21996, Fax: +942-122-22545

\begin{abstract}
Cesarean scar pregnancy is a rare form of ectopic pregnancy where the conceptus is implanted at the site of a previous cesarean section scar which pauses lifethreatening complications if not diagnosed and managed timely. Though numerous types of treatment approaches have been described there is no consensus on the optimal treatment modality which is a challenge to clinicians. Current treatment approaches are a combination of medical and surgical interventions with varying success rates and complications.

In this paper, we report a case of viable cesarean scar pregnancy classified as Type 1 with higher serum level of serum $\beta-h C G$ which was treated successfully by oral administration of mifepristone alone without the use of methotrexate followed by trans-cervical suction evacuation of the conceptus under real-time ultrasound guidance with minimal blood loss. Hemorrhage following evacuation being the commonest complication of this combined approach which is commonly managed with intrauterine folly catheter tamponade, our patient was treated with prophylactic intravenous tranexamic acid during evacuation warranting no further surgical interventions.

Given the successful outcome in our case with minimum morbidity, the option of oral mifepristone followed by suction evacuation with prophylactic use of tranexamic acid must be give consideration when a cesarean scar pregnancy presents with a higher level of serum $\beta-h C G$, a live fetus and where it is classified as Type-1.
\end{abstract}

\section{Keywords}

Cesarean, Ectopic, Mifepristone, Pregnancy, Scar, Tranexamic acid

\section{Introduction}

Cesarean scar pregnancy (CSP) is a rare form of ectopic pregnancy where the conceptus is implanted at the site of a previous cesarean section scar [1]. Its incidence was reported in the range of $1 / 1800$ to $1 / 2400$ of all pregnancies and represents $6 \%$ of all ectopic pregnancies in women with prior cesarean delivery $[2,3]$. Symptoms of cesarean scar pregnancy vary highly and majority of women may be asymptomatic [4]

Considering the rarity of such pregnancies and its serious and life-threatening complications, it is important to make an early and accurate diagnosis, plan treatment strategies to prevent serious complications and to preserve future fertility if possible $[5,6]$. CSP is also considered a precursor of morbidly adherent placenta and an expectant approach to this condition does not seems to be an option [7].

Though numerous types of treatment approaches have been described there is no consensus on the optimal treatment modality and CSP still is a challenge

Citation: Muhunthan K, Amirthanathan AJ, Raguraman S, Guruparan K (2021) Successful Treatment of a Cesarean Scar Ectopic Pregnancy Using a Novel Approach with Oral Mifepristone followed by Trans Cervical Suction Evacuation with Prophylactic Tranexamic Acid: A Case Report. Obstet Gynecol Cases Rev 8:211. doi.org/10.23937/2377-9004/1410211

Accepted: October 07, 2021: Published: October 09, 2021

Copyright: (C) 2021 Muhunthan K, et al. This is an open-access article distributed under the terms of the Creative Commons Attribution License, which permits unrestricted use, distribution, and reproduction in any medium, provided the original author and source are credited. 
to the clinicians [8].

Current treatment approaches are a combination of local and/or systemic administration of methotrexate, uterine artery embolization followed by dilatation and curettage or excision of trophoblastic tissues through laparotomy or laparoscopy [9]. Though methotrexate is the common medical therapy used in the combined management approach of CSP, cross-sectional analysis of this mode of treatment has showed its relatively low efficacy and higher complication rates including some serious side effects of systemic methotrexate [10].

Systemic methotrexate also has been shown to be less effective when the serum $\beta$-hCG levels are more than $5000 \mathrm{IU} / \mathrm{L}$ and the gestational age is more than 8 weeks [11].

In the event of a live fetus an anti-progestogenic agent namely mifepristone has been used in combination with methotrexate to shorten the time until embryos death and to reduce the dose of methotrexate but mifepristone alone has not been used in combined treatment approaches [12].

Mifepristone is a synthetic steroid with antiprogesterone effects on the endometrium and myometrium in women. This anti-progestational activity of mifepristone results from competitive interaction with progesterone at progesterone-receptor sites and inhibits the activity of endogenous or exogenous progesterone.

Life-threatening haemorrhage is a serious complication after evacuation of CSP and varied approaches have been recommended to arrest bleeding ranging from uterine artery embolization to intrauterine balloon tamponade with foley cater [9].
Though the use of intravenous tranexamic acid is recommended for the treatment of postpartum hemorrhage and has been shown to be safe and effective in trauma and surgery, with no apparent increase in vascular occlusive events $[13,14]$. The use of intravenous tranexamic acid has not been described in any reported cases of CSP.

In this paper, we report a case of viable cesarean scar pregnancy classified as Type 1 that was treated successfully by oral administration of mifepristone alone without the use of methotrexate followed by transcervical suction evacuation of the conceptus under real-time ultrasound guidance with a prophylactic intravenous tranexamic acid, warranting no further surgical interventions.

\section{Case Description}

A 34-year-old gravida 3 para 2, with two previous caesarean sections presented at a period of amenorrhea of 8 weeks and a positive urine pregnancy test.

Transabdominal and a transvaginal scans performed revealed an empty uterine cavity and empty endocervical canal. A gestational sac was located in the anterior wall of the isthmic portion, at the level of the previous caesarean section scar confirming a diagnosis of CSP. The myometrium between the gestational sac and the bladder was thin measuring only $3 \mathrm{~mm}$.

A live single fetal pole and a yolk sac were visible with a crown rump length of $12 \mathrm{~mm}$, equaling 7 weeks of gestation (Figure 1).

Based on our imaging this CSP was classified as Type1 or endogenic as the gestational sac was more towards the uterine cavity. There was also a $33 \mathrm{~mm}$ corpus

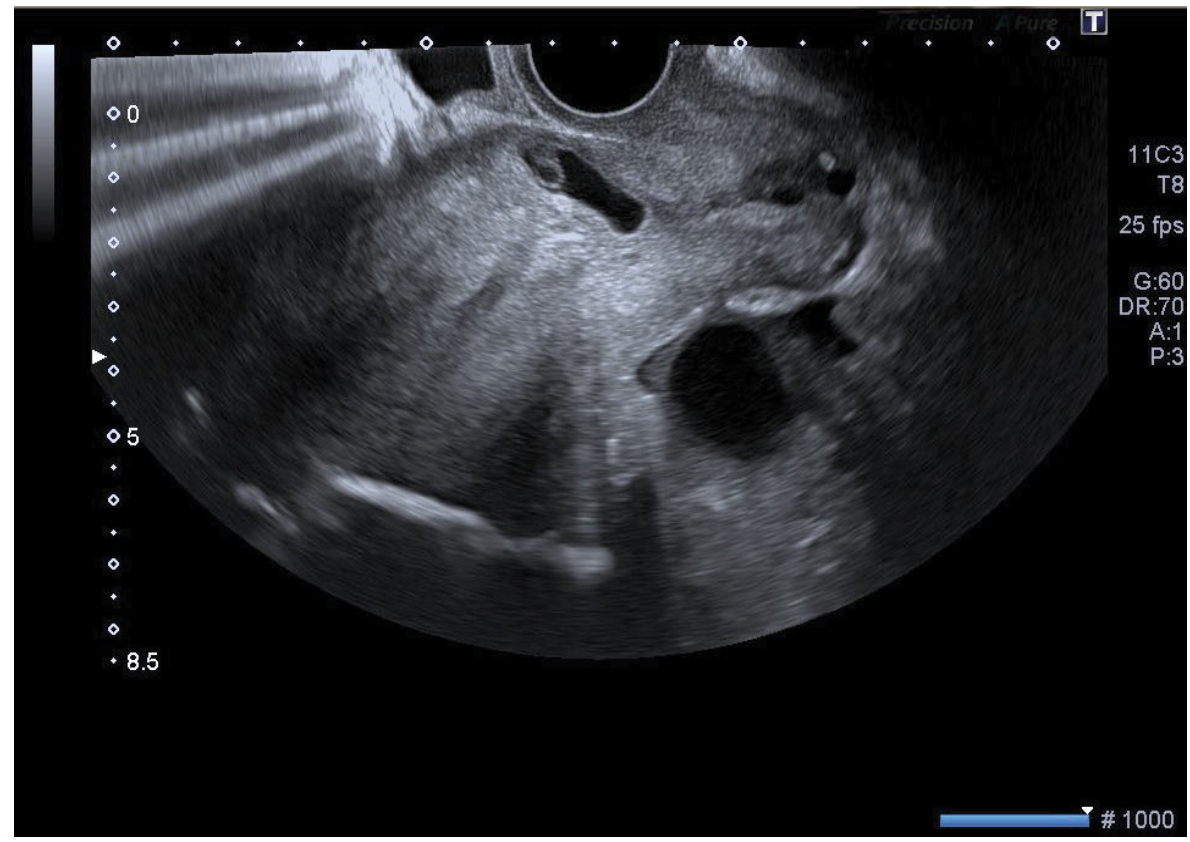

Figure 1: Transvaginal ultrasound image of the scar pregnancy with a fetal pole and yolk sac. A 33 mm corpus luteal cyst is seen in the cul de sac. 


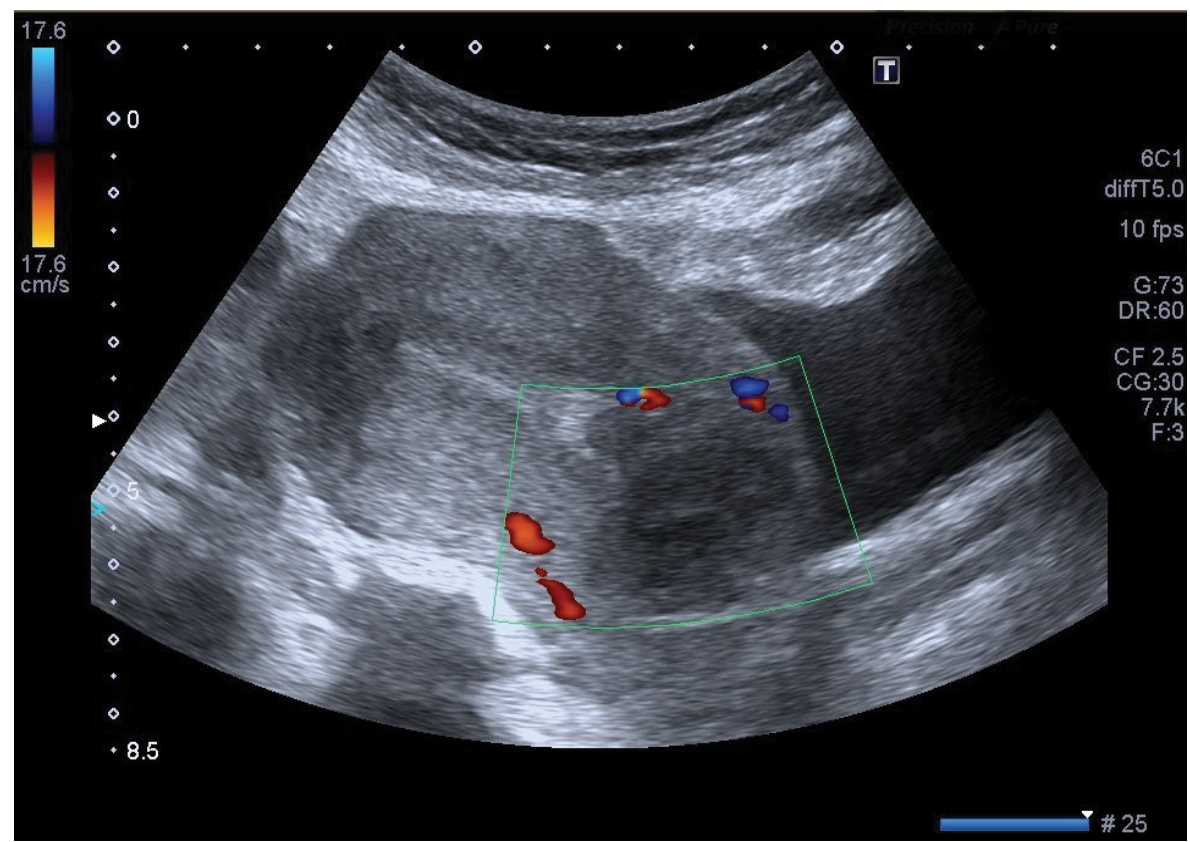

Figure 2: A follow up transvaginal ultrasound image performed 24 hours after the suction evacuation revealed $35 \mathrm{~mm}$ hemostatic clot occupying the site of her caesarean scar pregnancy.

luteal cyst on the right ovary and the pouch of Douglas was free of any fluid. A serum $\beta$-hCG performed at this moment was $16018 \mathrm{IU} / \mathrm{L}$ and the routine blood and urine investigations were normal.

Treatment options were discussed with the patient and in view of the higher serum $\beta$-hCG level and a live gestation decision was made to initially treat her with mifepriston orally with close monitoring.

She was initially treated with mifepristone $200 \mathrm{mg}$ orally. As the fetal viability was still present after 48 hours of the first dose, the same dose of mifepristone was repeated and the serum $\beta$-hCG performed at this moment was $21149 \mathrm{IU} / \mathrm{L}$.

She developed mild vaginal bleeding after the second dose of mifepristone. Administration of methotrexate was withheld as the patient declined its use considering the side effects.

After a clear discussion with the patient the need of laparotomy and a possible hysterectomy in the event of serious haemorrhage a transcervical suction evacuation of the conceptus was performed under real-time ultrasound guidance 72 hours after the initial administration of mifepristone.

She was administered tranexamic acid $1 \mathrm{~g}$ intravenously before the process of evacuation and the total estimated blood loss was $70 \mathrm{ml}$ not necessitating the use of intrauterine balloon tamponade.

The patient's vitals were closely monitored for 24 hours and she had an uneventful post-operative recovery.

A follow up ultrasound scan performed 24 hours after the suction evacuation revealed $35 \mathrm{~mm}$ hemostatic clot occupying the site of her caesarean scar pregnancy and the serum $\beta$-hCG had dropped to $4564 \mathrm{IU} / \mathrm{L}$ (Figure 2).

The patient was discharged home 24 hours after the evacuation as she was hemodynamically stable with minimal prevaginal bleeding. On a regular follow up two weeks after the evacuation she was asymptomatic and her $\beta$-hCG has dropped to undetectable levels and was prescribed with combined oral pills as contraceptive.

\section{Conclusion}

With the increasing awareness and clear ultrasonic criteria to diagnose cesarean scar pregnancy, early suspicion and diagnosis of this condition is possible with transvaginal ultrasound scan.

Transvaginal ultrasonographic diagnosis is made by visualization of a mass embedded in the hysterotomy scar, an empty uterine cavity, and thinning of a visible defect in the myometrium between the bladder and the sac.

Though a combined approach of management namely systemic methotrexate followed by suction evacuation seems to be the commonest approach of treatment, its low efficacy rates, higher complication rates including some serious side effects of systemic methotrexate and poor acceptance by patients deters its use certain patients.

Systematic reviews of its management approaches have failed to identify the leading method for treatment of cesarean scar pregnancy supporting the need for further studies in this field [8].

The anti-pregestational mifepristone has significantly lower side effect profile compared to methotrexate and use of this drug before the suction evacuation must 
be considered as a first line approach on such patient in whom systemic methotrexate is considered less effective.

Hemorrhage following evacuation is a recognised complication of this combined approach which was commonly managed with intrauterine folly catheter tamponade. Our decision to use intravenous tranexamic acid during evacuation was supported by its proven efficacy in the treatment of postpartum hemorrhage, trauma and surgery, with no apparent increase in vascular occlusive events.

Given the successful outcome in our case with minimum morbidity, the option of oral mifepristone followed by suction evacuation with prophylactic use of tranexamic acid must be give consideration when CSP presents with a higher serum level of hCG, a live fetus and where the CSP is classified as Type-1.

\section{Data Availability}

No data were used to support this study.

\section{Consent}

The patient has given written informed consent for the case to be published not disclosing her identity.

All information has been deidentified to preserve confidentiality.

\section{Conflicts of Interest}

The authors have no conflicts of interest.

\section{References}

1. Herman A, Weinraub Z, Avrech O, Maymon R, Ron-El R, et al. (1995) Follow up and outcome of isthmic pregnancy located in a previous caesarean section scar. $\mathrm{Br} \mathrm{J}$ Obstet Gynaecol 102: 839-841.

2. Rotas MA, Haberman S, Levsgur M (2006) Cesarean scar ectopic pregnancies: Etiology, diagnosis, and management. Obstet Gynecol 107: 1373-1381.

3. Seow KM, Huang LW, Lin YH, Lin MY, Tsai $Y L$, et al. (2004) Cesarean scar pregnancy: Issues in management. Ultrasound Obstet Gynecol 23: 247-253.
4. Olga G, Uma D, Linda F, Katherine K, Razeq A, et al. (2018) Cesarean scar pregnancy, incidence, and recurrence: Fiveyear experience at a single tertiary care referral center. Obstet Gynecol 132: 1285-1295.

5. Cignini P, Giorlandino M, Caserta L, Dominici L, Giorlandino C (2007) The importance of early diagnosis in Caesarean scar pregnancy. J Prenat Med 1: 29-31.

6. Collins K, Kothari A (2015) Catastrophic consequences of a caesarean scar pregnancy missed on ultrasound. Australas J Ultrasound Med 18: 150-156.

7. Timor-Tritsch IE, Monteagudo A, Cali G, PalaciosJaraquemada JM, Maymon R, et al. (2014) Cesarean scar pregnancy and early placenta accrete share common histology. Ultrasound Obstet Gynecol 43: 383-395.

8. Gonzalez N, Tulandi T (2017) Cesarean scar pregnancy: A systematic review. J Minim Invasive Gynecol 24: 731-738.

9. Jayaram PM, Okunoye GO, Konje J (2017) Caesarean scar ectopic pregnancy: Diagnostic challenges and management options. The Obstetrician \& Gynaecologist 19: 13-20.

10. Kanat-Pektas M, Bodur S, Dundar O, BakırVL (2016) Systematic review: What is the best first- line approach for cesarean section ectopic pregnancy? Taiwan J Obstet Gynecol 55: 263-269.

11. Maymon R, Halperin R, Mendlovic S, Schneider D, Herman A (2004) Ectopic pregnancies in a caesarean scar: review of the medical approach to an iatrogenic complication. Hum Reprod Update 10: 515-523.

12. Pristavu A, Vinturache A, Mihalceanu E, Pintilie $R$, Onofriescu M, et al. (2020) Combination of medical and surgical management in successful treatment of caesarean scar pregnancy: A case report series. BMC Pregnancy Childbirth.

13. CRASH-2 Collaborators, Shakur H, Roberts I, Bautista $\mathrm{R}$, Caballero J, et al. (2010) Effects of tranexamic acid on death, vascular occlusive events, and blood transfusion in trauma patients with significant haemorrhage (CRASH-2): A randomized, placebo-controlled trial. Lancet 376: 23-32.

14. WOMAN Trial Collaborators (2017) Effect of early tranexamic acid administration on mortality, hysterectomy, and other morbidities in women with postpartum haemorrhage (WOMAN): An international, randomized, double-blind, placebo-controlled trial. Lancet 389: 2105-2116. 